\title{
Vulnerabilidad y retribución: hacia una estética de la cuentística de Xavier Vargas Pardo ${ }^{1}$
}

\author{
Vulnerability and Retribution: in Search of the Aesthetics \\ of Xavier Vargas Pardo's Short Stories
}

\author{
Luis Miguel Estrada Orozco \\ Brown University \\ luis_miguel_estrada_orozco@brown.edu
}

Resumen: El libro Céfero (1961), de Xavier Vargas Pardo, permaneció desatendido por la crítica hasta años recientes. Las reseñas inmediatas a su publicación enfatizaron su particular uso del habla rural del Bajío mexicano, mientras que los enfoques contemporáneos han optado por la interpretación de los hechos violentos descritos en los once cuentos del volumen. A la par de esta violencia, en el volumen surge otra constante como correlativo necesario: la vulnerabilidad de sus personajes. El presente artículo propone una revaloración crítica del libro de Vargas Pardo a través del análisis de las condiciones de vulnerabilidad de los personajes sujetos y/o perpetradores de esta violencia, así como los actos de retribución por agentes no-humanos que ofrecen un balance inesperado ante la ausencia de justicia que domina en el libro del michoacano.

\author{
Palabras Clave: \\ Xavier Vargas Pardo; \\ Céfero; \\ cuento mexicano; \\ violencia; \\ vulnerabilidad.
}

\begin{abstract}
Xavier Varg
Céfero;

Mexican short story;

violence;

vulnerability.

ABSTRACT: The book Céfero (1961), by Xavier Vargas Pardo, remained unattended by critics until recent years. Reviews right after its publication emphasized its particular use of the rural speech from central Mexico, while contemporary approaches have favored interpretations of the violent deeds described in the eleven short stories of the volume. Next to this violence, another element in the book emerges as a necessary correlative: characters' vulnerability. This article offers a critical revalorization of Vargas Pardo's book through the analysis of the conditions of vulner-
\end{abstract}

1 El presente artículo retoma datos y consideraciones incluidas antes en "Céfero y sus rescatistas", en Cambiavías. Ciudad de México, núm. 6 (otoño de 2018). 
ability in the characters who are both subjects and/or perpetrators of the violence, as well as the acts of retribution by non-human agents who offer an unexpected balance to the absence of justice dominating the book from Michoacán.

recibido: 13 diciembre 2018

aceptado: 28 abril 2019

En un texto tan lúcido como emotivo, Edmundo Valadés recuerda el "descubrimiento" de Xavier Vargas Pardo (Tingüindín, Michoacán, 1923-Guadalajara, Jalisco, 1985) y de su único volumen de cuentos, Céfero, publicado por el Fondo de Cultura Económica en 1961. El objetivo de Valadés es rescatar del olvido el libro de Vargas Pardo, "para situarlo, como merece, entre nuestros mejores narradores" (1991: 64). Valadés no es escueto en sus apreciaciones y ofrece al lector una suerte de primer diagrama para los análisis críticos de Céfero a través de tres elementos: su riqueza lingüística, su buena manufactura técnica y los hechos "dramáticos o terriblemente violentos" que se detallan en los once cuentos del volumen (1991: 63-64). El presente artículo busca responder a esa invitación del rescate que propone Valadés a través de la revisión del único libro que el michoacano entregó a la imprenta; para ello, se centrará en los últimos dos elementos sugeridos por el sonorense: los elementos estructurales de los cuentos y los hechos violentos que se narran.

Los once cuentos que conforman el libro siguen las peripecias de Ceferino Uritzi, "Céfero", en el México rural de la Revolución y sobre todo durante los años inmediatamente posteriores a ésta. Si bien su primera recepción crítica destacó sus giros lingüísticos como un elemento dominante en la estética del libro, sus lecturas posteriores han buscado ahondar en las maneras en que la violencia se ha configurado como el "vínculo ambiguo e inmediato con los demás" para el hombre rural de Michoacán (Molina 1997: 7). Las implicaciones de esta violencia no descansan solamente en las razones por las que sus ejecutores son capaces de perpetrarla. Más allá de los bandidos que raptan mujeres, del violador y asesino que es ajusticiado en un aserradero o de los asaltantes que balean y apuñalan al narrador del libro, las lecturas sucesivas de la obra de Vargas Pardo lentamente 
dejan entrever una estética en la que la vulnerabilidad de los personajes se perfila como un elemento que vuelve una y otra vez a lo largo de su libro.

Así, proponemos una lectura de los cuentos de Xavier Vargas Pardo desde las distintas vulnerabilidades de los cuerpos violentados o en peligro inminente. Como se verá, los extremos de la violencia destacan las condiciones de vulnerabilidad, superpuestas a las condiciones económicas o de género de los personajes, o bien a la ausencia de un aparato de justicia. En un mundo propuesto donde reina la impunidad, algunos transgresores se convierten en víctimas de la violencia por virtud de agentes de retribución no-humanos como animales u objetos inanimados que el discurso presenta como "malditos". Los extremos a los que llegan las transgresiones y los castigos, o la desproporción del sufrimiento de los personajes, sólo consiguen poner de relieve las condiciones que vuelven vulnerables a los personajes en cuestión.

\section{Vargas Pardo: contexto y crítica}

En el texto antes señalado, Edmundo Valadés narra su breve participación como "padrino literario" de Vargas Pardo, además de enlistar algunos de los múltiples lectores por quienes el libro pasó antes de llegar a la prestigiosa colección "Letras mexicanas" del Fondo de Cultura Económica. Entre los lectores que recuerda Valadés destacan Gastón García Cantú, Rosario Castellanos y Juan Rulfo, quien, a decir de Valadés, evaluó la obra "de modo escueto pero muy expresivo. 'Éste se las sabe todas"" (Valadés 1991: 59).

Tanto la colección en que se incluyó al libro como el momento de publicación y sus lectores obligan a anotar algunas consideraciones, que además contextualizan la obra de Vargas Pardo en la literatura mexicana de su época. Por un lado, el año de publicación (1961) corresponde a un periodo de transición en el que la literatura urbana comienza a ganar preeminencia sobre la otrora dominante literatura con temas sobre el campo, la cual vio a varios de sus mejores expositores en la literatura sobre la Revolución mexicana (Brushwood 1985: 19). Por otro lado, sabemos que la fecha de escritura de estos cuentos es cercana a la publicación de obras esenciales escritas por algunos de sus lectores. Después de 
todo, "se trata de cuentos que escribió entre los años de 1954 y 1955" (El Nacional 1958).

La mención de Rulfo como uno de los lectores de Céfero trae a la mente los posibles puntos de contacto con la colección de cuentos decisiva de tema rural en la literatura mexicana, El llano en llamas (publicado en 1953), en particular a través de dos características que comparte con la obra de Vargas Pardo: su interés en el habla (con resultados notoriamente distintos) y una reiterada preocupación por la desposesión. Libros de cuentos más cercanos a la fecha de publicación de Céfero dan cuenta también de otras confluencias temáticas, como el estado de vulnerabilidad de miembros de comunidades históricamente marginadas. De nuevo, los lectores del material de Vargas Pardo dan buenos ejemplos de cuentística de esta línea. Tal es el caso de Ciudad Real, de Rosario Castellanos, publicado sólo un año antes de la aparición de Céfero. A pesar de que su principal diferencia es el énfasis de Castellanos en la cuestión indígena, no dejan de ser evidentes los vínculos que los unen, a través de estéticas que encaran el mundo de los desposeídos.

Un comentario crítico más hace pensar en las vinculaciones entre los temas tratados en Céfero y en los lectores involucrados de alguna manera en su publicación. Luis Leal, en su evaluación del cuento de la Revolución mexicana, recuerda que hacia 1947 éste ha desaparecido casi por completo. "Sin embargo, los neorrealistas -Córdova, Rulfo, Valadés, García Cantú, entre otros - siguen usando motivos revolucionarios" (Leal 2013: 327; cursivas nuestras). Como veremos, algunos "motivos revolucionarios" se mantienen presentes también en Vargas Pardo, quien acaso, cabría como uno de estos "neorrealistas" de los que habla Leal. A pesar de que no son dominantes, los temas revolucionarios otorgan sin duda al menos un marco de delimitación histórica y explican, si bien tangencialmente, algunas de las limitaciones en las vidas de sus personajes, además de su vinculación con un estado constante de violencia.

A pesar de que Céfero fue avalado por lectores cuyas cuentísticas tienen puntos de contacto con Vargas Pardo y, a pesar de insertarse en un contexto donde el material de la literatura con tema rural no ha desaparecido por completo, el libro tuvo "poca resonancia e incluso reservas y objeciones" tras su publicación (Valadés 1991: 60). Esta poca resonancia chocó de 
frente con lo que el propio Edmundo Valadés vaticinaba antes de la publicación del libro, cuando a finales de 1960 anticipaba que el michoacano sería "reconocido como uno de los más interesantes cuentistas de la vida rural, al aparecer su libro inédito" (1960: 29).

Para Valadés, el mérito más notorio es su manejo de los elementos lingüísticos propios del habla popular de Michoacán (1960: 29; 1991: 60). Este elemento lingüístico fue destacado por sus primeros reseñistas (Lerín 1961; Arellano 1961; Blanco 1962), a la vez que se probó como un obstáculo para la recepción más amplia de la obra. En entrevista, en 1965, el mismo autor michoacano reconoció las complicaciones de su obra de cara a una posible traducción (Flores 1965). Por otro lado, una recopilación antológica del cuento mexicano publicada en Montevideo, advierte que "Dios mediante" resulta difícil de leer (Raviolo 1980: 6) e incluso se dio a la tarea de ofrecer un breve glosario de los regionalismos que el antologador juzgó oscuros. ${ }^{2}$ Curiosamente, es un lector estadounidense quien opone un contrapeso a estas reservas al afirmar, en una reseña de 1961, que la ausencia de un conocimiento de regionalismos no debería ser obstáculo para que un lector competente disfrutara de la obra de Vargas Pardo (De Luca 1962: 184).

Los regionalismos, así como un tono y estilo consistentes, desembocan en un discurso en el que predomina la pertinencia lingüística del enunciante (Genette 1993: 84). Así, el discurso ubica a un narrador completamente inmerso en el mundo que describe: no narra lo que ocurre a los personajes, sino que lo vive con ellos desde dentro. Adicionalmente, este registro le permite a Vargas Pardo formar la base del persistente humorismo que hace el contrapunto con que los hombres del campo mexicano "se salvan o superan las incidencias desagradables, las injusticias, los azares de la vida, librándose de caer en el resentimiento o la amargura" (Valadés 1991: 61). Una mirada detenida a los cuentos de Vargas Pardo vuelve notorias las condiciones de donde esta injusticia surge. Sin embargo, antes de puntualizar las coordenadas de donde surgen la desposesión, aislamiento y ausencia de autoridades que dominan el mundo cerrado de

2 En realidad, los dos volúmenes del Panorama del cuento mexicano dan un tratamiento similar al léxico usado por otros autores incluidos como Juan Rulfo, Felipe Rojas González y Eraclio Zepeda, entre varios otros. Cfr. Vargas Pardo (1980: 16-25). 
Céfero, vale la pena hacer una apreciación final sobre las razones que nos llevan a analizar estos cuentos como un conjunto enlazado.

La estrategia que sigue Vargas Pardo construye a un solo narradorpersonaje-testigo (según el cuento en cuestión) que entrega al lector los once cuentos, a veces hablando desde su experiencia, a veces con funciones de un narrador que completa las historias "con ese gran ojo avizor de la vox pópuli" (González 2005: 70) o quizás desde la invención, convirtiéndose en una suerte de narrador omnisciente. La efectividad de este narrador hace que las entradas y salidas de su calidad de testigo y del punto de vista omnisciente se vuelvan menos relevantes para el lector que la efectividad que genera en la articulación de la historia. Por otro lado, el uso ininterrumpido del mismo narrador-personaje-testigo, así como la reincidencia de ambientes y temas, inserta el libro de Vargas Pardo en la tradición de lo que Russell M. Cluff Ilama el "cuento enlazado", donde las secuencias cuentísticas cumplen con cuatro características básicas: 1) los cuentos mantienen su autonomía, pero refuerzan entre sí contextos, temas, personajes o símbolos; 2) la secuencia se consigue a través de estrategias textuales como el título y/o elementos tales como narradores, ambientes, temas y personajes; 3) los cuentos adquieren coherencia por contrapunto, yuxtaposición o secuencias temporales, y 4) se trata de un conjunto armado intencionalmente por un mismo autor, no por un editor o antologador (Cluff 2013: 640). El mundo coherente de Céfero, así, no depende exclusivamente de su voz, sino también de su estrategia textual que entrega un marco superior de contextualización y de interacciones simbólicas y estéticas reforzadas por varios de sus cuentos.

\section{Céfero, realidad y contexto}

Raúl Eduardo González sugiere que una biografía de Ceferino Uritzi, el narrador, es intrascendente desde el punto de vista literario (2005: 70). Algunos de sus argumentos se sostienen en que a pesar de que sabemos que Vargas Pardo tomó el nombre y algo del anecdotario de una persona real, un obrero textil que conoció en su propio trabajo, en Jalisco (Flores 1965), la obra es evidentemente un trabajo de ficción (González 2005: 
71). De cualquier modo, vale la pena puntualizar el momento histórico que contextualiza el periodo en el que se insertan los personajes de la ficción.

El punto más temprano de la biografía del narrador queda establecido en el penúltimo cuento del volumen, "Quince ahorcados en Jiquilpan", cuando Céfero identifica su propia edad: "Yo apenas tenía diecinueve años" (Vargas Pardo 2005: 110). Este cuento, uno de los mejores del libro, se ubica en un año impreciso cuando las únicas coordenadas son que "en ese tiempo de la Revolución la mala vida era igual pa todos" (2005: 110) y donde las figuras dominantes que asolan la región, el Bajío michoacano, son "unas veces el Chivo, otras Chávez García y otras Samaniego" (110). Las menciones de Luis Gutiérrez, conocido como el Chivo Encantado, Inés Chávez García y quien probablemente es Ignacio Samaniego, entregan un marco mínimo de referencia histórica. Los tres operaron en Michoacán, donde la violencia vinculada con la Revolución inició en 1911 y se recrudeció hacia 1915 (Garciadiego 2010: 834). El nombre más reconocible, sin duda, es el de Inés Chávez García, dado que Michoacán, a diferencia de otros estados, "no proporcionó grandes contingentes a convencionistas, villistas o zapatistas, a pesar de lo cual no puede negarse el auge que tuvieron varios grupos anticarrancistas de la región, destacando el encabezado por [Inés] Chávez García" (2010: 834-35), entre cuyos aliados conocidos destacó Luis Gutiérrez, el Chivo Encantado (841). Ambos, por otro lado, murieron poco después de que se suscitó el punto más álgido del bandidaje en la región. El Chivo Encantado fue ejecutado y su cabeza fue exhibida en Colima en 1916 (Ochoa 2005: 104-105), mientras que Chávez murió víctima de la influenza española en 1918 (2005: 21).

La particularidad va más allá de la referencia temporal y entrega lo específico de la región. Gutiérrez y Chávez García ${ }^{3}$ se han mantenido en el imaginario popular no como figuras asociadas a sus supuestas lealtades políticas, sino debido a los excesos de su gente y el periodo extendido de bandidaje en que participaron como parte de las "hordas salvajes que se legitimaban en la Revolución" (Miranda 2006: 180). La reputación de esta

3 No es mi intención discutir la figura política de Chávez García en términos de villano o héroe de la Revolución. Para una discusión informada al respecto, el material referido de Álvaro Ochoa Serrano (2005) ofrece una excelente guía de fuentes. 
gente y su ausencia de programa político tienen un ejemplo en el segundo cuento del libro que nos ocupa, "El Churingo", cuyo título es el apodo que recibe un hombre de la zona michoacana de Tierra Caliente a quien Céfero conoce mientras trabaja como arriero: "Era un zaragate de primera y un asesino de mucho tiempo. Por allí contaban sus hazañas y que fue de los consentidos del Chivo Encantado cuando apenas tenía catorce años, porque una vez le rebanó los senos a dos muchachas pa que le dieran lo de más abajo" (Vargas Pardo 2005: 20; nuestras cursivas). Así, un nombre, un acto y una reputación bastan para dibujar en pocos trazos a un personaje que será devorado en la oscuridad de una mina abandonada.

Las siguientes coordenadas históricas relevantes del libro las otorgan el último cuento, "Dios mediante" y el primero, "Timbiriches en el cielo". "Dios mediante" sugiere que la acción se ubica después de que Lázaro Cárdenas se posicionara como una figura política, pues uno de los personajes asegura haberle hecho unos zapatos "a mi general Lázaro Cárdenas" (Vargas Pardo 2005: 135). La mención permite inferir que el cuento se ubica en cualquier momento posterior a la elección de Cárdenas como gobernador de Michoacán, en 1928, puesto que inició la parte más visible de su carrera política hasta su presidencia en el sexenio 1934-1940 (Meyer y Sherman 1991: 575-576). "Timbiriches en el cielo", por su parte, se ubica en un ambiente que sugiere urbanidad e industrialización. Ceferino, después de todo, trabaja como albañil "en la construcción de la fábrica con el gringo ese Linsi" (Vargas 2005: 7). Este cuento es el único en el que se lee un ambiente urbano desde la óptica de un antiguo hombre de campo. Sabemos, además, que para entonces ya no es un muchacho de diecinueve años, sino que está casado y que acaba de nacer su tercer hijo, "el más tiernito: Eliberto" (2005: 9). La excepcional urbanidad del primer cuento del volumen pone de relieve un elemento más: la vida del hombre de campo que necesita adaptarse a su nueva vida urbana, una tendencia demográfica notoria que había comenzado en las décadas de 1920 a 1940 (Meyer y Sherman 1991: 587-588) y que continuó con el énfasis industrial de la década de 1940 en adelante (1991: 611-614).

Así, el arco general de la vida de Ceferino Uritzi, y con ella, del mundo en el que viven las historias del volumen queda delimitado. Se trata de un mundo mayormente rural en Michoacán, en un periodo que inicia 
en algún punto cercano a 1915, en plena Revolución, que pasa por el problema del campo empobrecido y con tierras en conflicto (el tema de "Dios mediante") y que se extiende hasta los procesos de urbanización y movilidad poblacional de la década de 1940 y los años posteriores. En este contexto es donde los personajes del libro se enfrentan, a través de diversas situaciones, a modos de violencia que exponen las condiciones que los hacen vulnerables.

\section{La emergencia del cuerpo vulnerable: la autoridad ausente y la justicia inoperante}

"Timbiriches en el cielo", desde su excepcionalidad urbana, establece uno de los primeros elementos dominantes del volumen: la vulnerabilidad del individuo desposeído y su impotencia frente a la violencia ante un sistema de justicia inoperante. El narrador, Ceferino Uritzi, es emboscado, recibe un disparo en el cuello y cuando finge estar muerto es apuñalado por uno de sus asaltantes, quienes buscan asegurarse de su muerte y, finalmente, le roban sus pocas posesiones. No es esta la primera vez que Ceferino ha enfrentado tal violencia: "Tres veces había estado a la orilla del pozo y con ésa la cuarta" (Vargas Pardo 2005: 9). Mientras el personaje agoniza en el hospital, sufre un proceso alucinatorio que lo convence de encontrarse en el tránsito hacia el mundo posterior a la muerte, aunque ese mundo en nada se asemeja a sus expectativas: "tampoco había ningún pormenor de lo que acá nos cuentan de la gloria. Porque, según malicié, ya habíamos Ilegado y me extrañó que ni puerta, ni San Pedro" (2005: 12). Ante la sospecha de que su condición de pobre también es un impedimento en la vida ultraterrena y ante la decepción que le produce el cielo, prefiere volver a su vida terrenal. Al recobrar la conciencia y comenzar a mejorar, los agentes del Ministerio Público se acercan a tomarle declaración, ante lo cual detalla la identidad de su asaltante, al cual parece conocer: "De eso hace como diez años... y dicen los de la autoridá que están ahora en el Juzgado que todavía lo andan buscando" (16).

Las primeras condiciones de vulnerabilidad, marginalidad y pobreza quedan patentes. Del mismo modo surge uno de los primeros temas del libro: la impotencia de los sujetos como Céfero ante la ausencia de un 
aparato de justicia funcional. En cuentos sucesivos, diversos personajes tendrán pasados violentos que parecerán no haber sido nunca juzgados por las autoridades. En algunos casos, debido a la propia violencia de los hechos alrededor de la Revolución y del bandidaje posterior en la zona de Michoacán. En algunos otros, debido a las mismas condiciones de inoperancia del aparato de justicia mexicano. Una mirada detenida a dos cuentos más del volumen da cuenta de esta situación; además, nos acerca a la manera en que la superposición de la vulnerabilidad opera en todo el libro.

"Dios mediante", de nuevo, propone a un sujeto vulnerable por sus condiciones socioeconómicas frente a un atacante, al menos en el sentido simbólico, frente al cual la justicia no hace nada. El cuento combina dos tiempos narrativos. El primero, la mirada de Céfero contemplando a su padrino "Leovigildo Teco que agarró el camino con su tambache de reliquias por el lomo" (Vargas Pardo 2005: 132). El segundo, recordando las razones por las que el padrino Teco camina dejando atrás el terreno que ha sido propiedad de su familia por generaciones "desde antes de haber Casa de Tenencia y tener uso de razón los tatas de los tatas más viejos del pueblo" (2005: 137). Los terratenientes locales Chuy Tereja y Juan Charol, ambos descritos como personas de ética ausente, han tratado de apoderarse de la propiedad del padrino Teco por años a través de todos los medios, al grado de que Céfero ha pensado en comunicarle a la autoridad del pueblo "cómo cada año le tumbaban la enramada del chayotal, y cómo le caparon los marranos de cría y le dejaron colgando el sobrante de limosna pa que se hiciera unas criadillas", además de que le habían alterado los linderos (136).

Justamente la demarcación más relevante para el cuento de Vargas Pardo es la del lindero con Juan Charol, a quien el padrino Teco le pide: "Pa este lado del fresno no se pase, Juan Charol, porque aquí descansan bajo tierra los güesitos de mi madre" (2005: 138). Con esta mención de la tierra como herencia materna, Vargas Pardo traza una línea con la lucha agraria zapatista, a pesar de la improbabilidad de que un decreto efectivamente zapatista se hubiera puesto en práctica en la región. ${ }^{4}$ Dice Juan Charol que la tierra le pertenece a Teco "desde el bandido ese, el tal Zapata que

4 Álvaro Ochoa Serrano, sin embargo, remite a la documentación de la comunicación con que Emiliano Zapata invitaba al ya mencionado cabecilla regional Inés Chávez García a unirse a su movimiento (2005: 51, 151-154). 
los hizo a ustedes dueños ahi nomás a la pura pendejada" (Vargas Pardo 2005: 138). A pesar del derecho que el padrino reclama, no tiene argumentos legales probatorios, pues éstos "se quemaron toditos en la Revolución cuando esos de la bola y hasta los sardos le metían cerillo a todo cuanto encontraban" (139). Las tensiones aumentan. Juan Charol no sólo tiene poder económico, sino también "los rifles treinta y los indios huécatos maloras, que mataban nomás por llenar la tripa o por un gruñido del siñor su amo" (136), los cuales sólo encuentran resistencia entre la gente de Chuy Chereja, al grado de que sus rencillas han dejado varios muertos en la región: "Y no hay castigo pa ninguno ni nadie que se anime a restirarles la rienda de a deveras" (143). La frase "Dios mediante" se repite a lo largo del cuento como una esperanza de justicia postergada y una verbalización de la vulnerabilidad y la impotencia: "Dios mediante", piensa Céfero, las autoridades intervendrán. "Dios mediante", le decía la madre a su hijo Leovigildo Teco, las cosas cambiarían. "Dios mediante", el padrino piensa que puede hallar una solución.

El cuento de Vargas Ilega al clímax cuando los "indios huécatos", ${ }^{5}$ borrachos, tiran lodo a las paredes recién blanqueadas de la casa de Teco y éste los obliga a retirarse diciéndoles: "¡Vayan a decirle a Juan Charol que mañana le vendo mi potrero a Chuy Chereja!" (2005: 143). Charol, enfurecido, lo amenaza y le asegura que "Juan Charol quita y pone al mero de la Tenencia y a los otros con quien tienes esperanzas" (144); echa así por tierra cualquier esperanza de recurrir a las autoridades frente a la represalia que no se hará esperar. Esa misma noche, en efecto, Juan Charol da la orden de "que desenterraran los restos de la difunta madre de mi padrino, que los pintaran de verde, blanco y colorado y los colgaran de una rama del fresno" (145). Cuando Céfero lo mira desaparecer, cargando los huesos de su madre, lamenta no haber podido ni siquiera ayudar a su padrino a despintarlos: "ya ni modo de rasparlos porque hubiera sido como mermarle a mi padrino Teco lo poco de madre que le quedaba" (146).

$\mathrm{Si}$ bien los sujetos de la violencia en ambos cuentos son sujetos vulnerables e impotentes debido a sus condiciones de marginalidad y pobreza

5 Originarios de Huécato, en el municipio de Chilchota, en el noroeste michoacano. 
(relativa, en el caso de "Dios mediante"), las condiciones de la ausencia de justicia presentan un contraste claro. Mientras que en el caso de "Timbiriches en el cielo" se trata de un crimen del fuero común, algo más complejo ocurre en "Dios mediante". En la pugna entre Chuy Chereja y Juan Charol lo que se disputa es el poder por medios violentos, y con el poder, también la autoridad. Hannah Arendt lo ponía de relieve ya al abordar el problema de la violencia. Su línea argumental propone un problema similar al que nos ocupa: la autoridad entre las sociedades se distingue por su reconocimiento público sin necesidad de coerción ni persuasión, mientras que la violencia se distingue por su carácter instrumental (Arendt 1970: 43); sin embargo, poder, autoridad y violencia son inseparables (1970: 44). El que sean inseparables, no significa que sean la misma cosa. Para Arendt, el poder "no necesita justificación: es inherente a la existencia misma de las comunidades. Lo que requiere es legitimidad" (48), la cual brota del actuar concertado y así se legitima, mirando hacia el pasado, al punto de concierto. La justificación, por otro lado, mira hacia un fin futuro. En este sentido, "la violencia puede ser justificable, pero nunca será legítima" (48). El caso de la disputa de tierras presenta este conflicto: el pasado que justifica el derecho de propiedad de Teco, su poder sobre la tierra tiene una justificación. La violencia en contra suya no es legítima de ningún modo. Más aún, las condiciones por las que Chereja y Charol ejercen poder y autoridad se basan únicamente en modos de coerción y violencia. Su capacidad de ejercicio y la inoperancia de figuras de autoridad reconocidas libremente es lo que enmarca la vulnerabilidad del sujeto empobrecido, el supuesto heredero de los triunfos de luchas agrarias y el primer y último desplazado por su fracaso. La vejación final de los restos de la madre apunta a la intención de Charol de violentar simbólicamente esa justificación del pasado. Su razón para pintar los huesos con los colores de la bandera mexicana se explica: "Pa que esté bien claro que la difunta le llamaba algo al bandido ese de Zapata" (Vargas Pardo 2005: 145). La vejación simbólica une el pasado familiar de Teco y también el momento supuestamente fundacional del cuerpo nacional moderno. Tanto los restos de la madre como el mismo padrino Teco son impotentes ante la violencia que se ejerce en contra suya; su vulnerabilidad es tal (y ha quedado patente durante el cuento) 
que no hace falta una violencia extrema directa, sino la pura exhibición simbólica de los restos maternos.

"Quince ahorcados en Jiquilpan" destaca como uno de los mejores cuentos del volumen. En este caso, los giros que ocurren en la trama ponen de relieve problemas similares entre el poder, la violencia y la autoridad a los que me he referido. Sin embargo, en este cuento hay más capas de análisis en lo que respecta a las vulnerabilidades superpuestas que emergen y, con ellas, las diferentes formas de impotencia.

La primera parte del cuento narra un amor condenado desde su nacimiento. Céfero labora como peón para don Filomeno Sosa, quien cultiva café. Céfero se enamora de Consolación, la hija de don Filomeno. El miedo a la inminencia de los peligros de la Revolución hace que él busque cortejarla a la brevedad; en sus propias palabras: "antes de que algún malora me la ganara o cualquier chico rato se la pepenaran los de la tropa" (Vargas Pardo 2005: 111). El medio con que Céfero elige conquistar a Consolación es notable. Se trata de una carta, un producto de lujo en su medio rural: "Costaban a dos por quinto las de primera [...] de modo es que yo me las tenía que arreglar con mis compas pa que me las escribieran de oquis" (2005: 110). La diferencia de clases y la capacidad del ejercicio de la violencia se vuelve pronto un problema. Tan pronto su relación se vuelve física, Céfero teme por su vida, pues tras su primer encuentro en el solar de la casa de Consolación, uno de los hermanos "que ya se las olía y me tenía mucha inquina cuando andaba grifo, entró al solar con mucho cuidado y con una retrocarga pa cairme a la descuidada" (112). El mundo de Céfero y Consolación pronto presenta una oposición, entre el mundo social y moral, y su mundo íntimo y trasgresor; una práctica, por otro lado, que no es exclusiva de ellos: "Así se estilaba con todas las muchachas de entonces, hasta con las apretadas y dizque de plaza" (115). Si bien reconocen la doble moral circundante, las intenciones de casarse de los amantes no flaquean por ello, ni son ajenos al doble problema que supone su situación. Días más tarde, en el mismo solar, discuten sus posibilidades de matrimonio, que dependen principalmente de la capacidad de Céfero de conseguir medios económicos. Independientemente de la posición de la familia de Consolación, ella misma, como mujer joven, no tiene capacidad económica y depende del hombre de la relación, el cual vive amenazado 
por los hombres de la familia de ella. A pesar de la pobreza de Céfero, Consolación queda subsumida a las capacidades productivas de éste, de cara a un matrimonio. Mientras tanto, el protagonista no puede oponerse a la familia de ella, siendo sujeto potencial de represalias. Mientras discuten este impase, ocurre el giro mayor del cuento: a lo lejos, Consolación escucha un ruido "¡Ahí vienen, Ceferino!" (Vargas Pardo 2005: 119).

A pesar de que en el cuento de Vargas los personajes se refieren a ellos como "los revolucionarios", se trata de la gente del Chivo Encantado. Consolación apenas logra ponerse un poco de ropa encima, Ceferino se esconde y don Filomeno y los hermanos de Consolación se presentan en el solar buscándola, pero nada pueden hacer contra ellos: "Uno lo atajó con el caballo [a don Filomeno] y le afocó el rifle, y el otro, al que le decían el Cuíjaro, se levantó a mi muchacha como si fuera de trapo y se la sentó en la cabeza de la silla" (2005: 120). La presencia de testigos les impide asaltar a Consolación y eligen llevarla "al jefe pa que la destutune a su gusto" (120). Céfero es incapaz de hacer nada, escondido como está, e incluso los hermanos "que ya traiban fajados sus cuetes" (121) son impotentes también. Como veíamos antes con Arendt, el poder surgido de la violencia emana directamente de su capacidad de ejercerla, de modo que los principales del pueblo, esa autoridad antes reconocible, queda desplazada. Incluso Ceferino participa de esta situación al referirse a la impotencia, la subyugación que él tiene con respecto a su patrón y éste con respecto a la gente del Chivo: "lo que no pudiera hacer mi patrón por Consolación no podía hacerlo nadie" (121). Esto se vuelve incluso más evidente cuando es el propio Chivo Encantado quien se presenta frente a la Presidencia Municipal, "mientras le juntaron a los del Ayuntamiento pa darles alguna encomienda o pa jodérselos de algún modo" (122; nuestras cursivas). La presencia del Chivo, en efecto, no busca desplazar de modo permanente a las autoridades; no busca sostener ninguno de los predicados que, según Walter Benjamin, le otorgan validez a la autoridad; es decir, su condición de "fundadora de derecho o conservadora de derecho" (Benjamin 1991: 32-33). Como hemos visto, no es ésta la función de Luis Gutiérrez, el Chivo Encantado. Su presencia en el pueblo se explica únicamente porque "le venían sacando a los federales y ya casi le pisaban los talones" (Vargas Pardo 2005: 122). No buscan comida, la cual rechazan, sino dinero. 
Su presencia en el pueblo es breve. Después de que el Cuíjaro le entrega a Consolación, el Chivo busca un préstamo forzoso tomando a diecinueve principales como rehenes, Consolación y don Filomeno incluidos. Manda a tres de ellos a reunir el dinero y los otros dieciséis quedan en custodia. Cuando le avisan que los federales vienen cerca, el Chivo sujeta a Consolación, la desnuda frente a todos y escribe en su trasero desnudo "Vale por $\$ 5,000$ pesos" (2005: 127). El Chivo y su gente toman camino hacia Jiquilpan, pero antes de salir manda a don Filomeno a que busque a la gente que ha debido recolectar ya el dinero y lo alcance. Le advierte que dejarán a cada uno de los rehenes colgados por el camino, de modo que si para cuando lleguen a Jiquilpan él no tiene el dinero "la garantía se termina y le dejo el documento colgado de un poste o de un mezquite" (128). El último tramo del cuento cierra con Céfero acompañando a don Filomeno a llevar el dinero del rescate. El ejército Ilega demasiado tarde, no tiene ningún momento para establecer el orden y, por lo que sabemos en el mundo de este cuento, no es mucho más confiable como institución que vele por la justicia.

En el camino a Jiquilpan, don Filomeno ha decidido no llevar a sus hijos sino a su empleado "porque tantiaba que a pesar de llevarles el dinero a los del Chivo también a nosotros nos iban a meter corte" (129). Bajo la lluvia y luego de "cuatro horas de miedo y sufrimiento" (130) en donde se debaten entre la esperanza de hallar a Consolación y la evidencia de catorce cuerpos colgados por el camino, al fin miran un pino de donde cuelga el cuerpo de la muchacha. Debajo de la inscripción que declara el valor de cinco mil pesos, el Chivo ha escrito "Pagado". Ceferino logra bajarla. "Y como la tenía boca arriba pa levantarla en peso, el letrero me quedó por debajo sobre una mano. Con el agua se fue reviniendo y empezó a escurrirme sobre el brazo, como si toda mi muchacha hubiera estado rellena con sangre de ese color morado" (131).

La crudeza del desenlace contrasta con la exaltación del primer tramo. En especial, la tinta como un sustituto de la sangre vuelve doblemente relevante la importancia de la palabra escrita en la conversión del cuerpo de Consolación. Si antes, para Ceferino, una carta la había convertido en la mujer amada, ahora los trazos de un lápiz-tinta la dejaban sólo como una garantía de pago que había perdido su valor y las cifras terminaban 
por disolverse en un proceso de muerte. En un libro donde el lenguaje es tan evidente como la violencia circundante, este detalle no es menor. Las implicaciones de lo que Ilama una "habilidad 'esenciante" son objeto de análisis para Slavoj Žižek, para quien en esta habilidad del lenguaje "existe una violencia fundamental: nuestro mundo sufre una torsión parcial, pierde su equilibrada inocencia, un color particular da el tono a la totalidad" (Žižek 2009: 87). La reducción particular del todo en el caso de Ceferino no es precisamente violenta, pero lo que ocurre con las palabras escritas en el cuerpo de Consolación resulta profundamente esenciante: convierte a esta mujer en un objeto sin más atributos que su capacidad de cambio.

La perspectiva de análisis de la violencia que Žižek privilegia tiene vinculaciones con el material que nos ocupa. Si bien existe una violencia objetiva, observable como la ejecución de Consolación, existe también una violencia subjetiva identificada con fuerzas sociales que es el verdadero origen de la violencia objetiva (2009: 19-25). Sin embargo, la violencia simbólica llega al punto del problema del "abismo del prójimo" en donde el otro es reducido a un concepto sesgado detrás del "muro del lenguaje" (92). El cuerpo de Consolación, su vulnerabilidad social y las palabras puestas sobre él para deshumanizarla y cosificarla, encarnan dolorosamente toda la violencia posible de su entorno, las cuales encuentran su extremo en la futilidad de su muerte, aunada a su vulnerabilidad extrema: es la sinrazón completa. Si cada uno de los otros personajes se encuentra en distintos grados de vulnerabilidad, es Consolación quien los enfrenta todos y es la palabra "Pagado" la evidencia última de ello.

\section{Vulneraciones y retribuciones: el balance de lo no-humano}

La vulnerabilidad superpuesta en los cuentos que hemos revisado hasta aquí se distingue por una impotencia constante ante la injusticia y el atropello. Como veíamos siguiendo a Arendt y a Benjamin, el mundo cerrado de Céfero incluye una agravante mayor en cuanto a que presenta la violencia como un cierto estado de sinrazón: no existe un poder que se establezca a través de ella. En los casos que hemos visto, hay un vacío, una 
autoridad en disputa y una ausencia de justicia que es de donde surge precisamente la vulnerabilidad de los personajes de Céfero revisados hasta aquí. En los tres cuentos descritos hay crímenes impunes. Sin embargo, el libro ofrece contrapuntos mediante cuentos en donde la ausencia de justicia es, en unos casos, paliada por lo que parece una presencia de elementos no-humanos que actúan como agentes de retribución. En otros casos, estos mismos agentes no-humanos imponen castigos excesivos cuando se suscitan trasgresiones menores, reforzando así las condiciones de vulnerabilidad manifiestas en el mundo de Céfero.

Al menos en un cuento ya mencionado aparece algo similar. En "El Churingo", el segundo cuento del libro, el trabajo de arriero lleva a Ceferino a Tierra Caliente, al sur de Michoacán, donde pasa "una temporada acarriando estiércol de murciélago de unos minerales que están cerca de Huetamo" (Vargas Pardo 2005: 18). Estos "minerales" o minas, constan de una red de túneles con "un montón de vericuetos de muchos kilómetros" (2005: 18) en cuyas paredes descansan los murciélagos chupasangre, "con las uñas y los dientes tan afilados como espinas de chicalote" (19). Ceferino y tres personas más, entre las que destaca el apodado Churingo, violador y asesino cuya fama ya hemos mencionado, se internan en ese laberinto de túneles. Durante su inmersión, las lámparas de carburo que llevan consigo se quedan sin agua, dejándolos sumidos en la total oscuridad y sin nada más que un par de cerillos. En su desesperación, el grupo agita a los murciélagos de la caverna, los cuales los atacan, cortándolos. El Churingo, con el torso desnudo, lleva la peor parte, pues además su cuerpo está infestado de niguas y liendres: "Con tantos brincos y tropezones le empezaron a sangrar las llagas de los dedos y los méndigos avechuchos le llovían por manadas" (21). Con el Churingo desangrándose por la ferocidad y cantidad de los ataques, el grupo continúa en un laberinto $\sin$ luz y, después de que ha pasado lo que sólo pueden suponer que es un día entero, acuerdan dejar a Ceferino atrás cuidando del Churingo, quien agoniza. Quedan así, en una oscuridad que Ceferino asegura "va a ser mi sepultura, muy amplia, eso sí" (23). En un momento final de iluminación, el Churingo orina dentro de la lámpara de carburo. Cuando Ceferino la enciende con el último cerillo, su primera visión, luego de dos días de oscuridad, es el cuerpo destrozado del Churingo agonizante: "Casi no tenía cara ni dedos porque se lo habían 
tragado los murciélagos. En cuanto lo destapé se quedó muerto. Y luego luego se le fue encima el animalero; lo taparon de la cintura pa'rriba [...] ¡comiéndoselo!" (24).

El ejemplo sucinto muestra el modo en que las trasgresiones pasadas encuentran un agente de retribución en el mundo natural o, por decirlo mejor, el mundo no-humano. En cierta manera, ponen de relieve una de las conexiones más sugerentes entre violencia y justicia que hace René Girard al repensar los sistemas judiciales desde una perspectiva que une la violencia y lo sagrado. Desde su óptica, la justicia opera como una forma socialmente sancionada (positivamente) de venganza pública. Girard supone que los sistemas religiosos de sociedades primitivas han dado origen a sistemas judiciales en las que el círculo vicioso de las represalias de la venganza implícita en la justicia se evita en tanto que la represalia es llevada a cabo por una instancia contra la cual no hay represalia posible; un sistema que funciona tanto mejor mientras menos conciencia se tenga de la vinculación directa de la venganza y la justicia (Girard 2005: 22-29). Esta idea sugiere al menos una explicación para el caso que nos ocupa. En otro sitio, Girard sugiere que en esta relación entre la violencia y lo sagrado, la violencia se percibe "como exterior al hombre; y ello se debe a que se funde y se confunde con lo sagrado, con las fuerzas que pesan realmente sobre el hombre desde fuera, la muerte, la enfermedad, los fenómenos naturales..." (90; cursivas nuestras). No es extraño, pues, que en el sistema de retribución bajo el que parece operar la forma de justicia que emerge en el cuento de Vargas Pardo haya una especie de sustitución sugerente entre una instancia sin posibilidad de represalias y un agente del mundo natural. En "El Churingo" no se enfatiza el abandono de los compañeros, sino el fin violento que cae sobre el Churingo. Ellos nunca vuelven y la retribución queda en manos de un agente natural que el narrador, quien comparte estos códigos idiosincráticos, destaca. Esta forma particular de justicia extrema por un agente no-humano ocurre en al menos dos casos más que vale la pena mencionar.

"Pancho Papadas" fue el primer cuento que le valió al autor algún reconocimiento, al ser premiado por la revista Perfumes y Modas, en 1958 (El Nacional 1958) y publicado en 1959 (Valadés 1991: 59). El relato narra la historia de un pequeño mono huasteco que Delfino, el "cuetero" (o espe- 
cialista en pirotecnia) del pueblo compra a un cilindrero para su esposa, Severa. Cuando ésta lo rechaza, el mono queda a merced de sus once hijos, célebres en el pueblo por sus travesuras, que bordean en lo criminal: "descalabraron al cura, le mataron la Picecua con todo y becerro a don Secundino, desenterraron los güesos del difunto Ponciano" (Vargas Pardo 2005: 72). Ellos, predeciblemente, lo torturan de diversos modos, hasta casi matarlo. Ceferino, vecino "casi puerta de por medio" (2005: 66), es un testigo impotente de todo esto. Sin embargo, cuando los niños tratan de ahogar a Pancho Papadas aventándolo "a las tinas de fermentar en la vinata de Huaquián" (73), Ceferino atestigua el cambio que se opera en el mono: "Algo le pasó desde aquella vez al huasteco. Como que no quedó en sus cabales, como que apenitas aguantaba esta vida, como que ya le daba lo mismo todo" (73).

El sufrimiento, su lugar marginal en el microcosmos del pueblo y la incapacidad de retribución o justicia, humaniza a Pancho Papadas. Como sugiere John Berger en su conocido ensayo "Why Look at Animals?", la primera relación del mundo humano con el mundo animal es metafórica (Berger 1980: 5); una metáfora que usa el simbolismo del animal para explicar lo humano (sea esto lo político, social o emotivo). Si bien Pancho Papadas se construye como un tropo que reúne en sí esa marginalización que Berger también observa en la gente de campo cercana al mundo animal (1980: 13), el mono no deja de pertenecer al mundo no-humano, capaz de generar retribuciones violentas. El final del cuento, de hecho, tiene un último gesto que vincula esta condición de "humanidad" ganada a través del sufrimiento vinculado a una forma retributiva de venganzajusticia propia de su condición de elemento no-humano (tal como ocurre en el universo de Vargas Pardo): Pancho Papadas retira deliberadamente la señal de peligro del barril de pólvora, ocasionando su estallido y la muerte de toda la familia del cuetero e incluso de "Ambrosio el guarachero que era compadre de Delfino y que andaba allí nomás de mirón" (Vargas Pardo 2005: 75).

"El anillo del zanate", por su parte, ofrece una visión más sobre las capacidades retributivas de lo no-humano y una curiosa subversión de las posibilidades del individuo vulnerable. Si bien Pancho Papadas actúa de manera directa, en "El anillo del zanate" la sortija que tiene la figura de esta ave 
tiene condiciones más bien de elemento propiciador. Tras alistarse como soldado en Matamoros, pues "no había más remedio, pa llenar la tripa" (2005: 32), Ceferino conoce a Camilio Guízar, un hombre taciturno que Ileva un anillo con la imagen de un zanate, y quien le explica que, a diferencia de las urracas, "los zanates son pájaros misteriosos [...] pero nunca hay que darles de comer, ni matarlos, ni quedárseles viendo, porque piscan el corazón de los hombres, envenenan y train la desgracia" (33). El anillo que trae, recuerdo de su mujer, quien le engaña para casarse, pues está embarazada de otro hombre, es un recuerdo de su tragedia personal. Al igual que las aves, "este anillo, Céfero, trai la desgracia a todo el que lo carga, a mí ya no, porque cuando mucho podría matarme y eso... ¡eso es lo que yo quisiera!" (34). En efecto, Camilio muere mientras la tropa defiende un tren de un asalto de bandidos (quizás vinculados con alguna facción revolucionaria, al menos de nombre) en la población michoacana de Ajuno (37). Este primer aviso de la muerte, además de las razones que han llevado a Ceferino y a Guízar a enlistarse, presenta un reverso sobre la vida del ejército. No se trata de hombres en posiciones de poder. Por el contrario, quienes deberían encarnar la máxima capacidad de daño no son mucho menos vulnerables que los propios atacantes a los que se enfrentan. Como veremos, no son mucho menos sujetos de protección de la justicia humana ni tienen mucha menos capacidad de escapar a las retribuciones de lo no-humano.

Ante el cadáver, el personaje apodado el Cortito toma la sortija a pesar de la advertencia de Céfero: "no hay que robar a los muertos" (37). Si bien la transgresión del Cortito es mucho menor que otros personajes que hemos visto, su fin no es mucho más agradable. El Cortito es un jugador compulsivo que "se la pasaba sobando la baraja y viendo a quién dejaba hasta sin mugre" (34). En la primera oportunidad que tienen de descansar tras la muerte de Camilio, el Cortito decide jugar a las cartas con "dos cuates fuereños" (38) a quienes trata de embaucar. Cuando uno de ellos apuesta demasiado y descubre el engaño del Cortito, quien lleva una carta escondida, decide castigarlo: "Más tardé yo en dar una parpadiada que él en levantar el machete y dejarlo cair sobre la canilla del Cortito. Pegó un grito y se fue de espaldas con todo y silla, con la punta del brazo echando sangre a cántaros" (39). La mano, por lo demás, queda sobre la mesa "con 
sus cinco dedos engarruñados, y en el más largo, el maldito anillo aquel, con su pájaro muy negro" (39).

El anillo presenta varios elementos aquí. Por un lado, un símbolo de la naturaleza, pero también de la desgracia posible. Por el otro, la desgracia, si se considera la manera en que el cuento está narrado, se percibe como inevitable. Incluso si el Cortito no conoce la historia del anillo y de Camilio, incluso si no sabe que el zanate es un ave de mal agüero, su comportamiento codicioso llega al punto de la transgresión cuando le roba a un muerto. Los tabúes vinculados con la muerte tienen un cierto eco en la manera de mirar la violencia como exterior al humano, que no es exclusivo de la visión de Girard. Para ello, basta recordar las palabras de George Bataille al revisar ciertas transgresiones: "Debemos huir de la muerte y ponernos al abrigo de las fuerzas desencadenadas que la habitan. No debemos dejar que en nosotros se desencadenen otras fuerzas análogas a aquellas de las que el muerto es víctima" (Bataille 2002: 51; cursivas nuestras). El cuento muestra precisamente la retribución que sobreviene luego de la trasgresión de ese ludópata tramposo que es el Cortito; su suerte se percibe como vinculada a la causalidad porque la narración se articula de ese modo. Es decir, queda la impresión de que lo mutilan por el robo al cadáver, más que por hacer trampa en la baraja. Por otra parte, es notable que del castigo a sus atacantes no haya ninguna mención. La justicia no opera así, ni siquiera para los soldados.

Finalmente, el cuento "Bembéricua" Ileva a un extremo brutal la serie de juegos de vulnerabilidades superpuestas que he detallado a la par de las condiciones de ausencia de justicia y de irrupciones de agentes no-humanos (o que al menos se perciben como tales). El cuento, en realidad, inicia con la descripción de un retablo, "pa quedar a mano con San Miguel", que "tiene pintadas unas matas de bembéricua" porque "fueron las que salvaron a la guare Cenobia" (Vargas Pardo 2005: 51). Así, toda la acción del cuento queda enmarcada en un contexto que involucra la conjunción de un elemento vegetal y la intervención divina por mediación angelical.

Al llegar a Tingüindín, Ceferino consigue trabajo en un aserradero acarreando troncos y conoce a los tres personajes principales del cuento: Nicho, Cenobia y el Criolina. Este último, al modo del Churingo, debe su fama a ser un célebre asesino de mujeres: "Ya lleva varias, dicen que 
seis o siete" (55). Aun cuando saben de su presencia en el pueblo, las propias autoridades no lo han detenido: "en este pueblo les llevó un cajón a la Presidencia, pa que tengan donde meterlo cuando se muera. Ya van dos veces que lo empacan porque amanece en cualquier esquina con la lengua de fuera, pero a la hora del entierro se levanta y él mismo se carga el cajón de vuelta..." (56). La condición de vulnerabilidad extrema se encuentra en Cenobia, una mujer indígena (siempre descrita como "guare", localismo para designar a las mujeres purépechas), que es la pareja de $\mathrm{Ni}$ cho, un trabajador del aserradero conocido por ayudar siempre a los otros. Si el Criolina es el asesino impune, Cenobia es una viuda embarazada que depende en gran medida de la protección que le brinda su condición de pareja de Nicho. Mientras el resto de los trabajadores se dedica a las actividades del aserradero, el Criolina embosca a la guare Cenobia, quien en un momento de desesperación invoca la protección del arcángel San Miguel $y$, acto seguido, se interna inadvertidamente entre matas de bembéricua (planta venenosa que causa escozor extremo, fiebres elevadas y riesgo de muerte). El Criolina, dado que sabe que no podrá concretar la violación, aguarda toda la noche en espera de que ocurra una de dos cosas: que Cenobia salga de las matas para matarla o que la intoxicación de la bembéricua acabe con ella.

El cuento alcanza su clímax precisamente cuando El Criolina pasa de ser victimario y se convierte en víctima. La propia narración de Céfero permite intuir el extremo de lo que va a ocurrir: "En el retablo ese del que hablaba más antes, el que le mandamos a hacer a San Miguel, ni de chiste está pintado lo que sigue aquí luego luego" (Vargas 2005: 64) Los trabajadores del aserradero, dirigidos por Nicho, sorprenden al Criolina, lo someten a punta de pistola y luego lo ejecutan cortándolo en dos con las herramientas del aserradero para, en un acto final de vejación, enterrar cada parte en un hoyo diferente: "no sea que se vuelva a enderezar cualquier chico rato" (65). En otro lugar, este acto ya ha sido analizado en los términos de una violencia ilegal que ilustra el fracaso de la búsqueda de la justicia, siguiendo las ideas tanto de Girard que hemos citado aquí, como las del Michel Foucault de Vigilar y castigar, entre otros (cfr. Estrada 2017). Lo que el presente artículo busca destacar, además de la teatralidad de una ejecución que empareja "al verdugo con un criminal y a los jueces 
con unos asesinos" (Foucault 1976: 16), son las condiciones de una vulnerabilidad que no es exclusiva del sujeto violentado, sino que también convierte al perpetrador de la violencia en una víctima potencial debido a sus condiciones de marginalidad. Es decir, la condición compartida tanto por los verdugos como los criminales es también su vulnerabilidad. Por otro lado, el esquema mental que supone el narrador del cuento, busca atribuciones divinas al castigo, aunque no logra evitar una sanción tan extrema como el acto que los ejecutores han perpetrado.

\section{Conclusiones}

En los cuentos analizados, los personajes de Céfero, en muchos casos, pasan de una posición de perpetradores (efectivos o potenciales) de la violencia a víctimas de ella o se posicionan como cuerpos marginales dentro de una sociedad ya de suyo marginal que padece por diversos factores a los que se atribuye su vulnerabilidad. Éstos tienen explicaciones en un marco histórico, en ocasiones, y en un entorno sociocultural, en otras. Si las vulnerabilidades de los personajes del mundo de Céfero se superponen de diversos modos, las retribuciones por elementos no-humanos enfatizan la fragilidad de la humanidad representada en Céfero. La voz que Vargas Pardo creó, ese narrador único que es Ceferino Uritzi, aparece en ocasiones como la voz del sujeto marginal que expone sus condiciones de vulnerabilidad. En otras, forma parte de una fábula admonitoria sobre las consecuencias de la marginalidad. En cualquier caso, el tono, la ironía y el humor configuran personajes cuyos mecanismos de resiliencia les permiten superar la fragilidad de la condición humana, siempre expuesta a la violencia.

\section{Bibliografía}

Arellano, Jesús. "Javier Vargas Pardo y Margarita Casasús", en El Nacional. Suplemento 745. México (9 de julio de 1961): 15.

Arendt, Hannah. Sobre la violencia. Trad. Miguel González. México: Joaquín Mortiz, 1970.

Batallle, Georges. El erotismo. Trad. Antoni Vicens y Marie Paule Sarazin. Barcelona: Tusquets Editores, 2002. 
Benjamin, Walter. Iluminaciones IV. Para una crítica de la violencia y otros ensayos. Introd. y selec. Eduardo Subirats. Trad. Roberto Blatt. Madrid: Taurus, 1991.

Berger, John. "Why Look at Animals?", en About looking. Nueva York: Pantheon Books, 1980. 1-26.

Blanco, Miguel. "Xavier Vargas Pardo, Céfero" (Reseña), en Anuario de Letras. México. Facultad de Filosofía y Letras de la Universidad Nacional Autónoma de México. II (1962): 300-301.

BrushwoOd, John S. La novela mexicana, 1967-1984. México: Grijalbo, 1985.

Cluff, Russell, M. "La historia de una tradición: el cuento enlazado en México", en Historia crítica del cuento mexicano del siglo Xx. Comp. Alfredo Pavón. t. II. Xalapa, Ver.: Universidad Veracruzana, 2013. 635-678.

El NaCional. "Recibió ayer su premio el ganador en el concurso de El Nacional", en El Nacional. México (30 de mayo de 1958).

Estrada Orozco, Luis Miguel. "Bembéricua: una interpretación de la violencia en la literatura de Xavier Vargas Pardo", en Amoxcalli. Puebla, Pue, vol. 2, núm. 3 (enero-julio, 2017): 51-68.

Flores, ERnesto. "Javier Vargas Pardo dijo...", en El Occidental. Guadalajara, Jal. (25 de febrero de 1965).

Foucault, Michel. Vigilar y castigar. Trad. Aurelio Garzón del Camino. México: Siglo XXI, 1976.

Garciadiego Dantán, Javier. "José Inés Chávez García, ¿rebelde, bandido social, simple bandolero o precursor de los cristeros?", en Historia Mexicana, vol. 60, núm. 2 (octubre-diciembre 2010): 833-895.

Genette, Gérard. Ficción y dicción. Trad. Carlos Manzano. Barcelona: Lumen, 1993.

GirARD, RenÉ. La violencia y lo sagrado. Trad. Joaquín Jordá. Barcelona: Anagrama, 2005.

González, Raúl Eduardo. "Realidad y ficción en Céfero, de Xavier Vargas Pardo", en Phillippe Schaffhauser y Blanca Cárdenas Fernández (comp.). La problemática de la identidad en la producción discursiva de América Latina. Memorias del X Coloquio Internacional de Literatura. Perpignan: Université de Perpignan, Centre de Recherches Ibériques Latino-Américaines, 2005. 69-76.

LEAL, LuIS. "La Revolución mexicana y el cuento", en Alfredo Pavón (comp). Historia crítica del cuento mexicano del siglo Xx, t. I. Xalapa, Ver.: Universidad Veracruzana, 2013. 327-363.

Lerín, Manuel. "Céfero", en El Nacional. Suplemento 745. México (9 de julio de 1961): 15.

LuCa De, A. Michael. "Céfero by Xavier Vargas Pardo" (Reseña), en Books Abroad. Oklahoma. Board of Regents of the University of Oklahoma, vol. 36, núm. 2 (primavera, 1962): 184.

Meyer, Michael C. y William L. Sherman. The Course of Mexican History. 4a. ed. Nueva York: Oxford University Press, 1991.

Miranda, Francisco. "Inés Chávez, muerto. Dos textos del padre Esquivel". Relaciones. Estudios de Historia y Sociedad. Morelia, Mich., vol. XXVII, núm. 105 (2006): 178-202. 
Molina García, Arturo. "Los cuentos de Xavier Humberto Vargas Pardo", en El Centavo. Revista de Cultura y Literatura. Morelia, Mich., año XLIII, núm. 211 (febrero 1997): 7-8.

Ochoa Serrano, Álvaro. Chávez García, vivo o muerto. Morelia, Mich.: MorevaIlado Editores, 2005.

Raviolo, Heber (sel. y pról.) Panorama del cuento mexicano (2). Montevideo: Ediciones de la Banda Oriental, 1980.

Valadés, Edmundo. "El cuento mexicano reciente", en Armas y Letras. Monterrey, N. L. (1960): 19-31.

Valadés, Edmundo. "Al rescate de Céfero", en Alfredo Pavón (ed.). Cuento de nunca acabar (la ficción en México). Tlaxcala, Tlax.: Universidad de Tlaxcala, 1991. 59-64.

Vargas Pardo, Xavier. Céfero [1961]. México: Fondo de Cultura Económica, 2005.

Vargas Pardo, Xavier. "Dios mediante", en Panorama del cuento mexicano (2). Selec. y pról. Heber Raviolo. Montevideo: Ediciones de la Banda Oriental, 1980. 16-25.

ŽıŽEK, SLAVOJ. Sobre la violencia: seis reflexiones marginales. Trad. Antonio José Antón Fernández. Barcelona: Paidós, 2009.

Luis Miguel Estrada Orozco

Postdoctoral Research Associate in International Humanities for the Cogut Institute, en Brown University. Doctor en Lenguas y Literaturas Romances por la Universidad de Cincinnati y Maestro en Literatura Mexicana por la Benemérita Universidad Autónoma de Puebla. Su investigación doctoral explora la figura del boxeador mexicano en la literatura del país, vinculándolo con representaciones de cine y prensa, además de discusiones intelectuales en torno a la formación del carácter nacional. Sus líneas de investigación incluyen las exploraciones de la violencia en la literatura mexicana pos-revolucionaria y estudios sobre masculinidades y narrativas nacionales en Latinoamérica. Como narrador, ha obtenido varios premios nacionales de cuento y ha sido incluido en antologías en México y España. 